\title{
Myelolipoma: A Rare Incidentally Detected Adrenal Lesion
}

\author{
Ashumi Gupta ${ }^{1 *}$, Mahendra Singh Punia ${ }^{2}$, Devendra Sharma² and Udai Beniwal ${ }^{2}$ \\ ${ }^{1}$ Department of Pathology, CK Birla Hospital, Jaipur. India \\ ${ }^{2}$ Department of Urology, CK Birla Hospital, Jaipur. India
}

\begin{abstract}
Adrenal myelolipomas are benign, uncommon neoplams that are being increasingly detected due to frequent use of imaging studies. They are lipomatous tumours, that are usually asymptomatic and sometimes associated with endocrinological dysfunction.

We present a case of middle aged gentleman, with accidentally detected adrenal mass and no hormonal disturbances. The patient was evaluated initially for fever. Laboratory investigations revealed evidence of urinary tract infection, which was conservatively managed. Ultrasound abdomen showed a hyperechoeic mass in suprarenal region with a hypoechoeic component. On further work up, MRI showed a well defined suprarenal mass with hyperintensity, possibly adrenal myelolipoma. The tumour was removed laparoscopically and histopathology revealed features of myelolipoma. The patient was discharged after an uneventful postoperative period.

Adrenal myelolipomas are rare, benign tumours of adrenal gland diagnosed incidentally. Careful evaluation is important including imaging studies and endocrinological testing. Larger or symptomatic tumours can be excised surgically. Laparoscopic resection is a safe procedure in tumours considered for surgery, with favourable patient outcome.
\end{abstract}

\section{Keywords: Adrenal Myelolipomas, Lipomatous Tumours, Benign, Rare}

\section{Introduction}

Adrenal myelolipomas are unusual, benign tumours of adrenal gland that are usually asymptomatic.There has been an increase in detection of these lesions due to more frequent use of imaging techniques. ${ }^{[1]}$ Majority of these tumours are diagnosed incidentally although some may present with varied symptoms. ${ }^{[2]}$ Histologically, they are composed of an admixture of lipomatous component and haematopoetic component, in varying proportions. We report an unusual case of adrenal myelolipoma, incidentally discovered in a 56 year old gentleman.

\section{Case report}

A 56 year old gentleman presented with fever, generalized weakness, and frequency of micturition. He had a past history of hypertension and type 2 diabetes mellitus, controlled on medication. The patient had undergone surgery for inguinal hernia 6 years back. There was no history of any drug allergy. General and systemic examination showed no positive findings. The patient was mildly hypertensive with blood pressure of 140/90 $\mathrm{mmHg}$.

Routine investigations showed presence of pus cells in urine, mild anaemia with borderline leukocytosis on blood counts. ESR was elevated. Malarial parasite antigen test was negative. Blood glucose, serum electrolytes and liver enzymes were within normal range. Tests for viral markers were non reactive. Urea and creatinine levels were mildly elevated $(41.8 \mathrm{mg} / \mathrm{dl}, 1.4 \mathrm{mg} / \mathrm{dl}$ respectively). $\mathrm{HbAlc}$ was borderline ( $6.6 \%$ ). ECG did not show any significant abnormality, apart from sinus tachycardia. Urine routine examination revealed pus cells 25-30/hpf. Urine culture showed Escherichia coli with significant colony count, and blood culture showed Escherichia coli.

On USG, Right kidney showed a hyperechoic oval shaped lesion measuring $6.8 \times 5.7 \mathrm{~cm}$ in upper pole with $2.4 \times 2 \mathrm{~cm}$ hypoechoic area within it, most likely adrenal mass lesion. Rest of abdominal organs including urinary tract did not show any significant abnormality. Patient was managed conservatively with medications for urinary tract infection. He improved symptomatically and blood counts came to normal. After six days, the patient was discharged.

Meanwhile further investigations were done to ascertain the nature of suprarenal lesion. Endocrine workup revealed serum aldosterone, serum cortisol and plasma free metanephrines within normal range. ( $\mathrm{S}$ aldosterone 3.24 $\mathrm{ng} / \mathrm{dl}$, Plasma free metanephrine $50 \mathrm{pg} / \mathrm{ml}$ (normal $<65$ ); serum cortisol $17.1 \mu \mathrm{g} / \mathrm{dl}$ )

MRI whole abdomen showed a large well-defined solid looking altered signal intensity mass lesion measuring $65 \times 52 \times 55 \mathrm{~mm}$, appearing slightly hyperintense on $\mathrm{T} 1$ 
images with loss of signal intensity on fat suppression images (Figure 1) in right supra renal gland. The lesion was abutting the inferior surface of liver with slight displacement of IVC. There was no significant abnormality in the urinary tract, no obvious pelvic or abdominal lymphadenopathy. On CECT there was a well circumscribed oval mass lesion, measuring $65 \times 67 \mathrm{~mm}$ in right renal upper pole in region of adrenal gland with soft tissue and fat; density -40 to +34 HU, with minimal punctate enhancement on contrast administration, most likely to be adrenal myelolipoma.

One month later the patient was taken up for laparoscopic adrenalectomy after routine investigations which did not show any abnormal findings. At that time, the patient was afebrile with general and systemic examinations not revealing any abnormality. There was no complaint of pain in abdomen or fever. His blood counts and urea/creatinine were normal.
Right laparoscopic adrenalectomy was done. Post operative stay was uneventful and the patient was discharged next day with follow up advice. At one month follow up, the patient was asymptomatic.

Pathological Findings: Gross examination revealed grey yellow, capsulated soft tissue measuring 6 × 5 x $5 \mathrm{~cm}$. Cut surface was grey yellow with haemorrhagic areas (Figure 2). On histopathological examination (Figure 3), sections showed a tumor composed of lobules of mature adipocytes, with focal fat necrosis. Focal haemorrhage and scattered foci of hematopoeitic elements including erythroid precursors, few myeloid cells and occasional megakaryocytes were seen. Normal adrenal gland tissue was present in the periphery. There was no capsular breach or any other evidence of malignancy in the sections examined. Based on these findings, a diagnosis of adrenal myelolipoma was given.

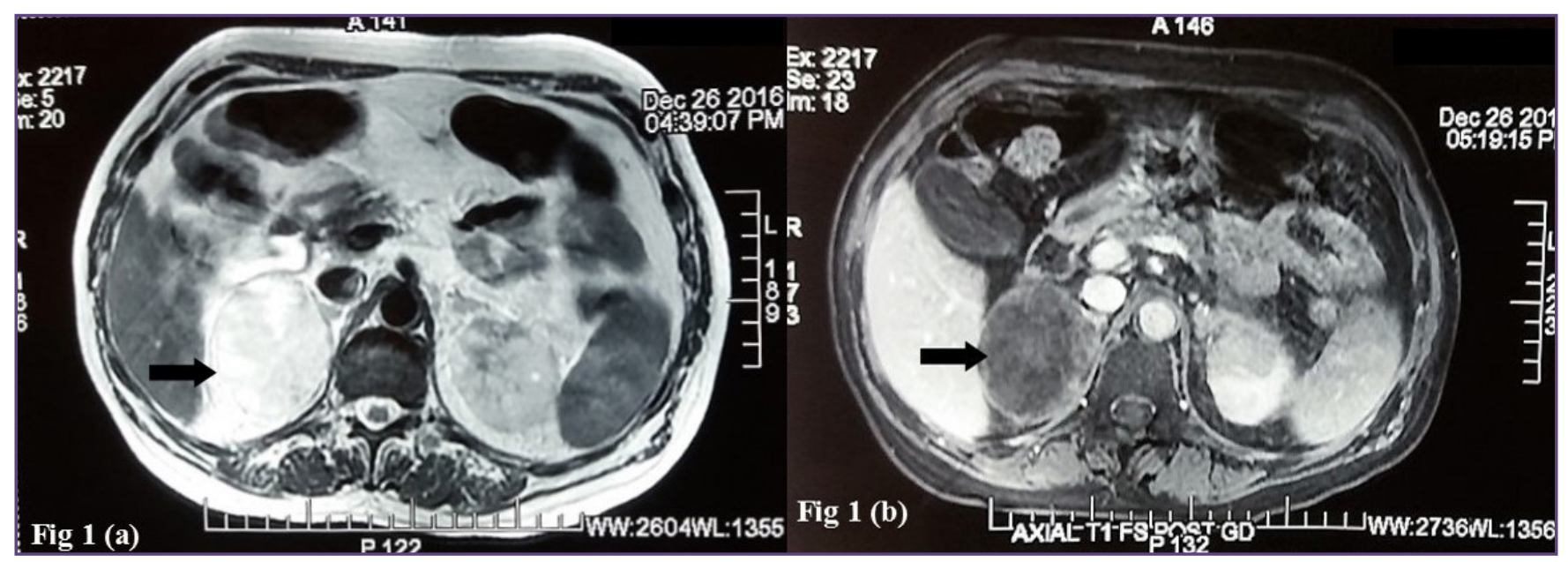

Fig. 1 (a) : MRI features of myelolipoma - hyperintense mass (arrow) in right suprarenal in T1 image.; (b): MRI features of myelolipoma - Loss of signal intensity in the lesion (arrow)with fat suppression on T1 MRI.

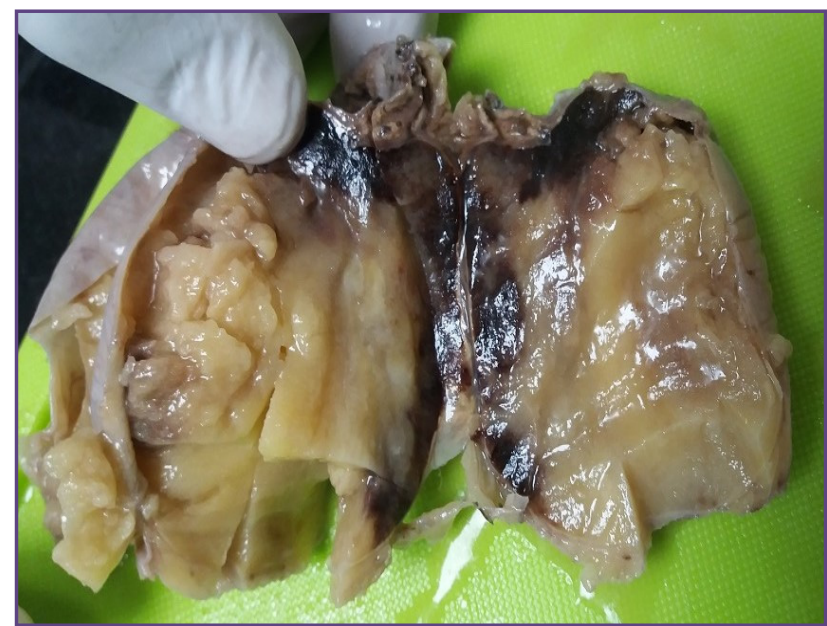

Fig. 2: Gross specimen showing encapsulated fat rich lesion with variegated areas.

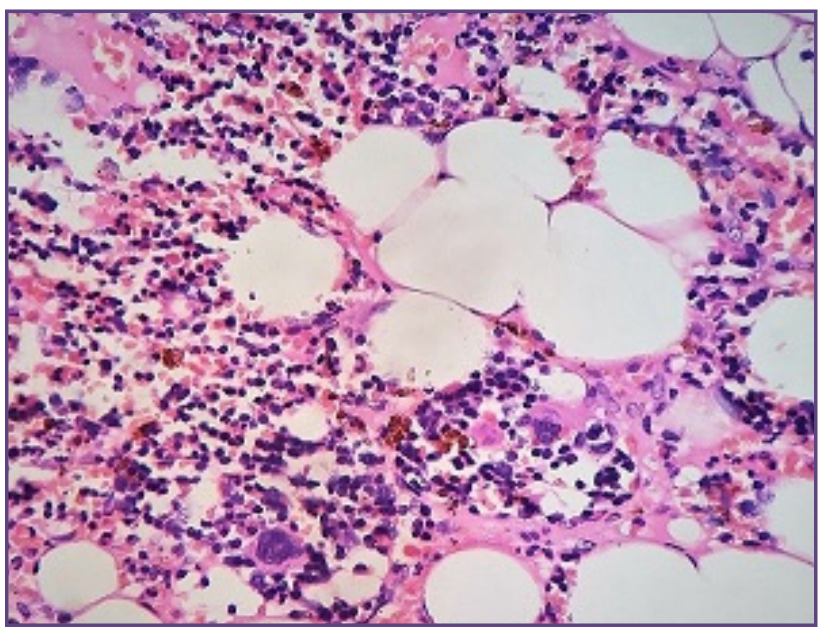

Fig. 3: H\&E 40x showing adipose tissue admixed with trilineage haematopoeisis including megakaryocytes. 


\section{Discussion}

Adrenal myelolipomas are rare tumours, constituting $2.6-6 \%$ of primary adrenal tumours. ${ }^{[2-4]}$ They are being reported more frequently due to increase in use of imaging investigations. ${ }^{[1,3]}$ They may be detected as an incidental finding in imaging studies, done for other reasons. [3] Sometimes adrenal myelolipoma may be symptomatic, presenting with pain, palpable abdominal mass, hypertension, endocrine disorders. ${ }^{[3,5,6]}$ Large tumours may rupture, leading to intraabdominal haemorrhage and may present with shock. ${ }^{[6]}$

Adrenal myelolipoma may be associated sometimes with endocrinological disturbances such as hyperaldosteronism, hypercortisolism, or hyperandrogenemia. There may be associated clinically apparent hormonal imbalance due to adrenocortical dysfunction in some cases, and aldosterone hypersecretion may be induced by some factor released by adrenal myelolipoma or due to pressure effects on the adjacent gland by tumor. However, further observation is required to investigate the association of endocrinological imbalances with adrenal myelolipoma. Compression of the surrounding tissue by the tumour may also lead to adrenocortical abnormalities in some cases. There may be associated hyperplasia of adrenal gland which may result in endocrinological disorder. ${ }^{[6]}$ Endocrinological work up may be helpful in excluding functional myelolipomas. Gershuni et al recommend evalulation of fractionated plasma metanephrines to exclude pheochromocytoma before proceeding for surgery. Cortisol evaluation and further hormonal workup may be considered depending on the clinical scenario. [2] Our case was clinically asymptomatic and the patient did not show any evidence of endocrinological abnormality on investigations.

Imaging techniques may be the first to point towards an incidental adrenal tumour. Adrenal myelipomas can be radiographically detected as well circumscribed lipomatous lesions with heterogenous density. ${ }^{[1-3]}$ Adrenal myelolipoma shows heterogenous echogenicity on ultrasonography. ${ }^{[7]}$ CT imaging indicates the suprarenal location, and shows characteristic appearance with a well circumscribed lesion with heterogeneous enhancement containing a fatty component. ${ }^{[3,7]}$ On Magnetic resonance imaging there is increased signal intensity in fatty areas on $\mathrm{T} 1$ weighted images. T2 weighted images show moderate hyperintensity admixed with medium signal intensity due to presence of haematopoetic elements. [1] Fat suppression images allow better characterisation of lesion. ${ }^{[8]}$ Myelolipomas are the most commonly encountered lipomatous tumours of adrenal gland. Rarely, they have been reported in the mediastinum, thorax, kidney and presacral region. ${ }^{[9-12]}$
Other lipomatous tumours that occur in adrenal include teratoma, lipoma, angiomyolipoma and liposarcoma. [3] Adrenal adenoma and adrenal carcinoma may also enter the differential diagnoses based on imaging findings, and it may not always be possible to make a definitive diagnosis on imaging in cases with lesser amount of fat component. [13]

On pathological examination, adrenal myelolipomas are macroscopically encapsulated with presence of fatty areas and tan brown areas, sometimes with haemorrhagic foci. ${ }^{\text {[14] }}$ Size may vary from small subcentimeter tumours to large masses upto $21 \mathrm{~cm}$. ${ }^{[2,3]}$ Microscopically they are composed of adipocytes and haematopoetic elements in varying proportions..$^{[1,13]}$ Adjacent adrenal tissue is usually evident. ${ }^{[1,14]}$ Larger tumours may show haemorrhage, necrosis, cystic change, calcification, ossification. ${ }^{[14]}$

For large tumours $(>=6 \mathrm{~cm})$ or symptomatic tumours, surgical excision may be performed. ${ }^{[14]}$ Smaller and asymptomatic tumours may be managed with careful patient follow up. Increase in size and atypical radiological features may be considered when making a clinical decision. ${ }^{[2,14]}$ Recently, laparoscopic adrenalectomy has been shown to be an effective methodology for excision of adrenal myelolipoma with lesser blood loss, shorter duration of post-operative stay. ${ }^{[13]}$ In our case, succesful laparoscopic excision of the adrenal tumour was performed without any complications and uneventful recovery of the patient. Laparoscopic adrenalectomy is a safe approach with favorable outcome in patients where surgical removal is indicated. ${ }^{[2,13]}$

\section{Conclusion}

Adrenal myelolipomas are unusual, benign adrenal lesions that are may be detected as an incidental finding following imaging studies. Characteristic imaging findings may allow a presumptive diagnosis. Careful work up is recommended including hormonal evaluation before making a clinical decision. Surgical excision may be considered in larger tumours, to prevent development of future complications. Laparoscopic surgery is an effective modality with good patient outcome and minimal complications.

\section{References}

1. Shenoy VG, Thota A, Shankar R, Desai MG. Adrenal myelolipoma: Controversies in its management. Indian $\mathrm{J}$ Urol. 2015. Apr-Jun; 31(2): 94-101.

2. Gershuni VM, Bittner JG Ivth, Moley JF, Brunt LM. Adrenal Myelolipoma: Operative Indications and Outcomes. J Laparoendosc Adv Surg Tech A. 2014 Jan;24(1);8-12.

3. Lam KY, Lo CY. Adrenal lipomatous tumours: a 30 year clinicopathological experience at a single institution . J Clin Pathol 2001;54(9):707-12. 
4. Song JH, Chaudhary FS, Mayo-Smith WW. The incidental adrenal mass on CT : prevalence of adrenal disease in 1049 adrenal masses in patients with no known malignancy. Am J Roentgenol. 2008 May;190(5): 1163-8.

5. Nabi J, Rafiq D, Authoy FN, Sofi GN. Incidental detection of adrenal myelolipoma : A case report and review of literature. Case Rep Urol. 2013. Epub 2013 Feb 20.

6. Su HC, Huang X, Zhou WL, Dai J, Huang BX, Cao WL, Sun FK. Pathologic analysis, diagnosis and treatment of adrenal myelolipoma. Can Urol Assoc J. 2014 Sep; 8 : 9-10.

7. Sandoval MA, Anel-Quimpo J. A giant myelolipoma discovered as an adrenal incidentaloma: radiological, endocrine and pathological evaluation. BMJ case Rep Dec 20;2010.

8. Ilias I, Sahdev A, Reznek RH, Grossman AB, Pacak K. The optimal imaging of adrenal tumours: a comparison of different methods. Endocr Relat Cancer. 2007 Sep;14(3):587-99.

9. Shi Q, Pan S, Bao Y, Fan H, Diao Y. Primary mediastinal myelolipoma: a case report and literature review. J Thorac Dis. 2017 Mar;9(3):E219-E225.
10. Shen C, Zhou K, Lai Y, Fan J, Liu L, Che G. Review of primary extra-adrenal myelolipoma of the thorax. J Surg Res. 2017 Jan;207:131-137.

11. Ghaouti M, Znati K, Jahid A, Zouaidia F, Bernoussi Z, Mahassini N. Renal myelolipoma: a rare extra-adrenal tumour in a rare site: a case report and review of the literature. J Med Case Rep. 2013 Apr 4;7:92.

12. Lee JJ, Dickson BC, Sreeharsha B, Gladdy RA, Thipphavong S. Presacral myelolipoma : Diagnosis on imaging with pathologic and clinical correlation. AJR Am J Roentgenol. 2016 Sep;207(3):470-81.

13. Yamashita S, Ito K, Furushima K, Fulushima J, Kameyama S, Harihara Y. Laparoscopic versus open adrenalectomy for adrenal myelolipoma. Ann Med Surg (Lond). 2014 Apr $16 ; 3(2): 34-8$.

14. Yalagachin GH, Bhat BK. Adrenal incidentaloma does it require surgical treatment? Case report and review of literature. Int J Case Rep. 2013;4(2):192-4.

*Corresponding author:

Dr. Ashumi Gupta, Department of Pathology, CK Birla Hospital, Jaipur, Rajasthan, India. 301018.

Phone: +91 8800921354

Email: ashumi.gupta@rbhri.in

Date of Submission : 29.03.2017

Date of Acceptance : 08.07.2017

Financial or other Competing Interests: None.
Date of Publication : 26.10.2017 